\title{
Bilinear Equations and Bäcklund Transformation for Generalized Ultradiscrete Soliton Solution
}

\author{
Hidetomo Nagai1, Daisuke Takahashi2 \\ Faculty of Science and Engineering, Waseda University, 3-4-1, Okubo, \\ Shinjuku-ku, Tokyo 169-8555, Japan
}

\begin{abstract}
Ultradiscrete soliton equations and Bäcklund transformation for a generalized soliton solution are presented. The equations include the ultradiscrete KdV equation or the ultradiscrete Toda equation in a special case. We also express the solution by the ultradiscrete permanent, which is defined by ultradiscretizing the signature-free determinant, that is, the permanent. Moreover, we discuss a relation between Bäcklund transformations for discrete and ultradiscrete $\mathrm{KdV}$ equations.
\end{abstract}

\section{Introduction}

Soliton equation has explicit $N$-soliton solutions and an infinite number of conserved quantities generally. In the beginning of the development of the soliton theory, continuous or semi-discrete soliton equations were studied mainly. For example, the Korteweg-de Vries (KdV) equation is a continuous soliton equation of PDE type, and the Toda equation is a semi-discrete soliton equation with continuous and discrete independent variables. There are two types of soliton solutions to the bilinear equations derived from these equations. One is expressed by a sum of a finite number of exponential functions, which was first proposed by Hirota 1, 2. We call this type of expression Type I. The other is expressed by Wronski determinant [3, 4. We call this type of expression Type II.

After the discovery of various continuous or semi-discrete soliton equations, discrete soliton equations of which independent variables are all discrete were proposed [5, 6]. Discrete soliton equation is also transformed into the bilinear equation and has multi-soliton solutions. It has also two types of expressions, Type I and II, where the determinant of Type II is generally the Casorati determinant for discrete soliton equations.

Discretization process is completed if dependent and independent variables are all discretized. In the 1990s, Tokihiro et al. proposed the ultradiscretization method to discretize dependent variables [7. The key formula in the method is

$$
\lim _{\varepsilon \rightarrow+0} \varepsilon \log \left(e^{a / \varepsilon}+e^{b / \varepsilon}\right)=\max (a, b) .
$$

Usual addition, multiplication and division for the real values in the original discrete equation are replaced with max operation, addition and subtraction respectively by this method. Due to these replacements, dependent variables can be discrete in the ultradiscrete equation if we use appropriate constants and initial values. Many ultradiscrete soliton equations or cellular automata have been proposed and the integrability is shown even for the digitized equations $[8,9$.

However, the operation in the ultradiscrete equation corresponding to the subtraction in the discrete equation is not well-defined. Thus we can not ultradiscretize a discrete equation automatically. This obstruction is called a 'negative problem' 10, 11. Thus the above soliton solution

\footnotetext{
${ }^{1}$ e-mail hdnagai@aoni.waseda.jp

2 e-mail daisuket@waseda.jp
} 
of Type II can not be ultradiscretized directly since the antisymmetry is crucial for the determinant. On the other hand, the solution of Type I can be ultradiscretized generally choosing the appropriate parameters included in the solution.

The imbalance between the two types of expression for the ultradiscrete soliton solution is partially solved. One of the authors (Takahashi) and Hirota proposed the ultradiscrete analogue of determinant solution for the ultradiscrete $\mathrm{KdV}(\mathrm{uKdV})$ equation 10 . One of the authors (Nagai) proposed the similar type of solution for the ultradiscrete Toda (uToda) equation[11]. This analogue is called an 'ultradiscrete permanent' (UP) defined by

$$
\max \left[a_{i j}\right] \equiv \max _{\pi} \sum_{1 \leq i \leq N} a_{i \pi_{i}}
$$

where $\left[a_{i j}\right]$ denotes an arbitrary $N \times N$ matrix and $\pi=\left\{\pi_{1}, \pi_{2}, \ldots, \pi_{N}\right\}$ an arbitrary permutation of $1,2, \ldots, N$. The $N$-soliton solution to the ultradiscrete bilinear equation of $\mathrm{uKdV}$ equation is expressed by the following two forms,

$$
f_{i}^{n}=\max _{\mu_{j}=0,1}\left(\sum_{1 \leq j \leq N} \mu_{j} s_{j}(n, i)-\sum_{1 \leq j<j^{\prime} \leq N} \mu_{j} \mu_{j^{\prime}} a_{j j^{\prime}}\right)
$$

and

$$
\tilde{f}_{i}^{n}=\frac{1}{2} \max \left[\begin{array}{cccc}
\left|s_{1}(n-N+1, i)\right| & \left|s_{1}(n-N+3, i)\right| & \ldots & \left|s_{1}(n+N-1, i)\right| \\
\ldots & \ldots & \ldots & \ldots \\
\left|s_{N}(n-N+1, i)\right| & \left|s_{N}(n-N+3, i)\right| & \ldots & \left|s_{N}(n+N-1, i)\right|
\end{array}\right]
$$

where $\max _{\mu_{j}=0,1} X\left(\mu_{1}, \mu_{2}, \ldots, \mu_{N}\right)$ denotes the maximum value of $X$ in $2^{N}$ possible cases of $\left\{\mu_{1}, \mu_{2}, \ldots, \mu_{N}\right\}$ replacing each $\mu_{j}$ by 0 or 1 . We call the form (3) Type I and (4) Type II respectively in this article.

Bäcklund transformation is an important object in the soliton theory since it gives the links among equations or solutions [5, 6]. The ultradiscrete version of the Bäcklund transformation is discussed in [12] or [13. The equations treated in the references are the ultradiscrete KadomtsevPetviashvili equation and the $\mathrm{uKdV}$ equation.

In this article, we consider a generalized solution of both types,

$$
f_{i}^{n}=\max _{\mu_{j}=0,1}\left(\sum_{1 \leq j \leq N} \mu_{j} s_{j}(n, i)-\sum_{1 \leq j<j^{\prime} \leq N} \mu_{j} \mu_{j^{\prime}} r_{j}\right)
$$

and

$$
\tilde{f}_{i}^{n}=\max \left[\begin{array}{cccc}
\left|s_{1}+(-N+1) \frac{r_{1}}{2}\right| & \left|s_{1}+(-N+3) \frac{r_{1}}{2}\right| & \ldots & \left|s_{1}+(N-1) \frac{r_{1}}{2}\right| \\
\ldots & \ldots & \ldots & \ldots \\
\left|s_{N}+(-N+1) \frac{r_{N}}{2}\right| & \left|s_{N}+(-N+3) \frac{r_{N}}{2}\right| & \ldots & \left|s_{N}+(N-1) \frac{r_{N}}{2}\right|
\end{array}\right],
$$

where

$$
\begin{aligned}
& s_{j}=s_{j}(n, i)=p_{j} n-q_{j} i+c_{j}, \quad 0 \leq p_{1} \leq p_{2} \leq \cdots \leq p_{N}, \\
& r_{j}=k p_{j}+l q_{j}, \quad k, l: \text { non-negative constants. }
\end{aligned}
$$

We discuss ultradiscrete soliton equations and a Bäcklund transformation for this solution.

The contents of this article are as follows. In Section 2, we give equations which $f_{i}^{n}$ and $\tilde{f}_{i}^{n}$ satisfy under some conditions. In Section 3, we show a Bäcklund transformation between $N$ and $(N+1)$-soliton solutions. In Section 4, we ultradiscretize the Bäcklund transformations for the discrete $\mathrm{KdV}$ equation and obtain those for the $\mathrm{uKdV}$ equation. The results suggests an algebraic 
correspondence between the solutions of determinant and of UP, In Section 5, we give concluding remarks.

The proofs in this article are given under the condition $p_{j} \geq 0$ for simplicity. However, note that these results can be easily extended to the case of arbitrary $p_{j}$ 's by replacing (5) with

$$
f_{i}^{n}=\max _{\mu_{j}=0,1}\left(\sum_{1 \leq j \leq N} \mu_{j} s_{j}(n, i)-\sum_{1 \leq j<j^{\prime} \leq N} \mu_{j} \mu_{j^{\prime}} a_{j j^{\prime}}\right)
$$

where

$$
a_{j j^{\prime}}=\min \left(\max \left(r_{j},-r_{j^{\prime}}\right), \max \left(-r_{j}, r_{j^{\prime}}\right)\right)
$$

\section{Bilinear Equations for the Generalized Soliton Solution}

First we give the following propositions.

Proposition 1 The generalized solution (5) with a dispersion relation

$$
q_{j}=\min \left(p_{j}, L\right),
$$

where $L(\geq 0)$ is a constant, satisfies a bilinear equation

$$
f_{i}^{n}+f_{i-l+1}^{n+k}=\max \left(f_{i-l}^{n+k-1}+f_{i+1}^{n+1}, f_{i-l}^{n+k}+f_{i+1}^{n}-L\right) \quad(k \geq 2) .
$$

Similarly, the following proposition holds.

Proposition 2 The generalized solution (5) with a dispersion relation

$$
q_{j}=\max \left(0, p_{j}-L\right)
$$

satisfies

$$
f_{i}^{n}+f_{i-l-1}^{n+k-1}=\max \left(f_{i-l}^{n+k-1}+f_{i-1}^{n}, f_{i-l}^{n+k}+f_{i-1}^{n-1}-L\right) \quad(k, l \geq 1) .
$$

Note that (11) reduces to the $\mathrm{uKdV}$ equation in the case of $(k, l)=(2,0)$, and (13) reduces to the uToda equation in the case of $(k, l)=(1,1)$ respectively.

Since the whole of proof of the propositions is long, let us show the outline. First, considering $f_{i+y}^{n+x}+f_{i+w}^{n+z}$ with arbitrary constants $x, y, z$ and $w$, we have from (5)

$$
\begin{aligned}
& f_{i+y}^{n+x}+f_{i+w}^{n+z}=\max _{\mu_{j}, \nu_{j}=0,1}\left(\sum_{1 \leq j \leq N}\left(\mu_{j}+\nu_{j}\right) s_{j}\right. \\
& \left.+\sum_{1 \leq j \leq N}\left(\mu_{j}\left(x p_{j}-y q_{j}\right)+\nu_{j}\left(z p_{j}-w q_{j}\right)\right)-\sum_{1 \leq j<j^{\prime} \leq N}\left(\mu_{j} \mu_{j^{\prime}}+\nu_{j} \nu_{j^{\prime}}\right) r_{j}\right)
\end{aligned}
$$

where $s_{j}$ denotes $s_{j}(n, i)$ for short. Using new parameters $\lambda_{j}$ and $\sigma_{j}$ defined by $\lambda_{j}=\mu_{j}+\nu_{j}$, $\sigma_{j}=\mu_{j}-\nu_{j}(1 \leq j \leq N)$, (14) reduces to

$$
\begin{aligned}
& f_{i+y}^{n+x}+f_{i+w}^{n+z} \\
= & \max _{\left(\lambda_{j}, \sigma_{j}\right)}\left(\sum_{1 \leq j \leq N} \lambda_{j} s_{j}+\frac{1}{2} \sum_{1 \leq j \leq N} \lambda_{j}\left((x+z) p_{j}-(y+w) q_{j}\right)\right. \\
& \left.+\frac{1}{2} \sum_{1 \leq j \leq N} \sigma_{j}\left((x-z) p_{j}-(y-w) q_{j}\right)-\frac{1}{2} \sum_{1 \leq j<j^{\prime} \leq N}\left(\lambda_{j} \lambda_{j^{\prime}}+\sigma_{j} \sigma_{j^{\prime}}\right) r_{j}\right) .
\end{aligned}
$$


Note that the pair $\left(\lambda_{j}, \sigma_{j}\right)$ can be one of the following,

$$
(0,0), \quad(1,1), \quad(1,-1), \quad(2,0),
$$

and $\max _{\left(\lambda_{j}, \sigma_{j}\right)} X\left(\lambda_{1}, \ldots, \lambda_{N}, \sigma_{1}, \ldots, \sigma_{N}\right)$ denotes the maximum value of $X$ in $4^{N}$ possible cases of $\left\{\lambda_{1}, \ldots, \lambda_{N}, \sigma_{1}, \ldots, \sigma_{N}\right\}$ replacing each $\left(\lambda_{j}, \sigma_{j}\right)$ by one of the above four pairs. Comparing the terms in (11) substituting (15), we can show that Proposition 1 holds if the following is proved for any $N^{\prime}$ satisfying $1 \leq N^{\prime} \leq N[10]$.

$$
\begin{aligned}
& \max _{\sigma_{j}= \pm 1}\left(\sum_{1 \leq j \leq N^{\prime}} \sigma_{j}\left(k p_{j}+(l-1) q_{j}\right)-\sum_{1 \leq j<j^{\prime} \leq N^{\prime}} \sigma_{j} \sigma_{j^{\prime}} r_{j}\right) \\
= & \max \left(\max _{\sigma_{j}= \pm 1}\left(\sum_{1 \leq j \leq N^{\prime}} \sigma_{j}\left((k-2) p_{j}+(l+1) q_{j}\right)-\sum_{1 \leq j<j^{\prime} \leq N^{\prime}} \sigma_{j} \sigma_{j^{\prime}} r_{j}\right),\right. \\
& \left.\max _{\sigma_{j}= \pm 1}\left(\sum_{1 \leq j \leq N^{\prime}} \sigma_{j}\left(k p_{j}+(l+1) q_{j}\right)-\sum_{1 \leq j<j^{\prime} \leq N^{\prime}} \sigma_{j} \sigma_{j^{\prime}} r_{j}\right)-2 L\right) .
\end{aligned}
$$

Using the dispersion relation (10), the maxima of LHS and of the first argument in RHS of (17) are both given by the case of $\sigma_{j}=(-1)^{N^{\prime}-j}\left(1 \leq j \leq N^{\prime}\right)$ [11. About the second argument in RHS, the case of $\sigma_{N^{\prime}}=1$ and $\sigma_{j}=(-1)^{N^{\prime}-j+1}\left(1 \leq j \leq N^{\prime}-1\right)$ gives the maximum. Therefore, (17) becomes

$$
\max \left(-p_{N^{\prime}}+q_{N^{\prime}}+p_{N^{\prime}-1}-q_{N^{\prime}-1}-p_{N^{\prime}-2}+q_{N^{\prime}-2}+\ldots, q_{N^{\prime}}-L\right)=0,
$$

after the above evaluation. In particular, the dispersion relation (10) derives

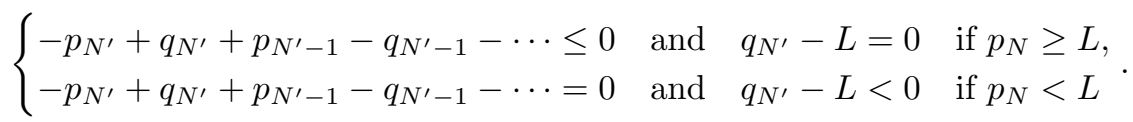

Thus (18) holds for any $1 \leq N^{\prime} \leq N$ and Proposition 1 is proved.

Comparing the terms in (13), we can show that Proposition 2 holds if the following is proved for any $1 \leq N^{\prime} \leq N$.

$$
\begin{aligned}
& \max _{\sigma_{j}= \pm 1}\left(\sum_{1 \leq j \leq N^{\prime}} \sigma_{j}\left((k-1) p_{j}+(l+1) q_{j}\right)-\sum_{1 \leq j<j^{\prime} \leq N^{\prime}} \sigma_{j} \sigma_{j^{\prime}} r_{j}\right) \\
= & \max \left(\max _{\sigma_{j}= \pm 1}\left(\sum_{1 \leq j \leq N^{\prime}} \sigma_{j}\left((k-1) p_{j}+(l-1) q_{j}\right)-\sum_{1 \leq j<j^{\prime} \leq N^{\prime}} \sigma_{j} \sigma_{j^{\prime}} r_{j}\right),\right. \\
& \left.\max _{\sigma_{j}= \pm 1}\left(\sum_{1 \leq j \leq N^{\prime}} \sigma_{j}\left((k+1) p_{j}+(l-1) q_{j}\right)-\sum_{1 \leq j<j^{\prime} \leq N^{\prime}} \sigma_{j} \sigma_{j^{\prime}} r_{j}\right)-2 L\right) .
\end{aligned}
$$

Using the dispersion relation (12), the case of $\sigma_{j}=(-1)^{N^{\prime}-j}\left(1 \leq j \leq N^{\prime}\right)$ gives the maxima of LHS and of the first argument in RHS. The case of $\sigma_{N^{\prime}}=1$ and $\sigma_{j}=(-1)^{N^{\prime}-j+1}\left(1 \leq j \leq N^{\prime}-1\right)$ gives the maximum of the second argument in RHS. Therefore, the above equation becomes

$$
\max \left(-q_{N^{\prime}}+q_{N^{\prime}-1}-q_{N^{\prime}-2}+q_{N^{\prime}-3}-\ldots, p_{N^{\prime}}-q_{N^{\prime}}-L\right)=0,
$$

after the above evaluation. This equation holds since

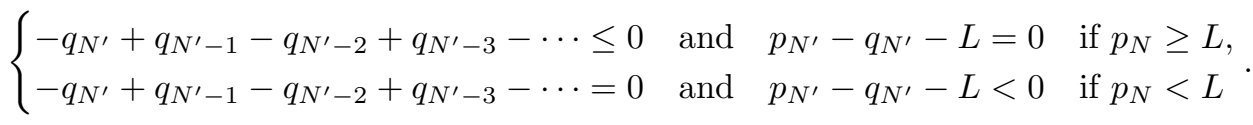

Thus Proposition 2 is proved.

Moreover these propositions lead the following propositions. 
Proposition 3 The generalized solution (6) satisfies

$$
\tilde{f}_{i}^{n}+\tilde{f}_{i-l+1}^{n+k}=\max \left(\tilde{f}_{i-l}^{n+k-1}+\tilde{f}_{i+1}^{n+1}, \tilde{f}_{i-l}^{n+k}+\tilde{f}_{i+1}^{n}-2 L\right)
$$

under the dispersion relation (10).

Proposition 4 The generalized solution (6) satisfies

$$
\tilde{f}_{i}^{n}+\tilde{f}_{i-l-1}^{n+k-1}=\max \left(\tilde{f}_{i-l}^{n+k-1}+\tilde{f}_{i-1}^{n}, \tilde{f}_{i-l}^{n+k}+\tilde{f}_{i-1}^{n-1}-2 L\right)
$$

under the dispersion relation (12).

Both propositions are proved by reducing (23) and (24) to (11) and (13) respectively. Using a formula[10]

$$
\begin{aligned}
& \max \left[\begin{array}{cccc}
\left|y_{1}+(-N+1) r_{1}\right| & \left|y_{1}+(-N+3) r_{1}\right| & \ldots & \left|y_{1}+(N-1) r_{1}\right| \\
\ldots & \ldots & \ldots & \ldots \\
\left|y_{N}+(-N+1) r_{N}\right| & \left|y_{N}+(-N+3) r_{N}\right| & \ldots & \left|y_{N}+(N-1) r_{N}\right|
\end{array}\right] \\
& =\max _{\rho_{j}= \pm 1}\left(\sum_{1 \leq j \leq N} \rho_{j} y_{j}-\sum_{1 \leq j<j^{\prime} \leq N} \rho_{j} \rho_{j^{\prime}} r_{j}\right)+\sum_{1 \leq j<j^{\prime} \leq N} r_{j^{\prime}} \text {, }
\end{aligned}
$$

where

$$
0 \leq r_{1} \leq r_{2} \leq \cdots \leq r_{N},
$$

and the transformation $\rho_{j}=2 \mu_{j}-1$, we have

$$
\begin{aligned}
\tilde{f}_{i}^{n}=\max _{\rho_{j}= \pm 1}\left(\sum_{1 \leq j \leq N} \rho_{j} s_{j}-\frac{1}{2} \sum_{1 \leq j<j^{\prime} \leq N} \rho_{j} \rho_{j^{\prime}} r_{j}\right)+\frac{1}{2} \sum_{1 \leq j<j^{\prime} \leq N} r_{j^{\prime}} \\
\approx \max _{\mu_{j}=0,1}\left(2 \sum_{1 \leq j \leq N} \mu_{j} s_{j}-2 \sum_{1 \leq j<j^{\prime} \leq N} \mu_{j} \mu_{j^{\prime}} r_{j}+\sum_{1 \leq j<j^{\prime} \leq N}\left(\mu_{j}+\mu_{j^{\prime}}\right) r_{j}\right) \\
\quad-\sum_{1 \leq j \leq N} s_{j}-\frac{1}{2} \sum_{1 \leq j<j^{\prime} \leq N} r_{j} \\
\approx 2 \max _{\mu_{j}=0,1}\left(\sum_{1 \leq j \leq N} \mu_{j}\left(s_{j}+\frac{N-j}{2} r_{j}+\frac{1}{2} \sum_{1 \leq j^{\prime} \leq j-1} r_{j^{\prime}}\right)-\sum_{1 \leq j<j^{\prime} \leq N} \mu_{j} \mu_{j^{\prime}} r_{j}\right) \\
-\sum_{1 \leq j \leq N} s_{j} .
\end{aligned}
$$

Here $f_{i}^{n} \approx g_{i}^{n}$ denotes that $f_{i}^{n}$ and $g_{i}^{n}$ give the same solution of (23) or (24). Hence, using a replacement

$$
c_{j}+\frac{N-j}{2} r_{j}+\frac{1}{2} \sum_{1 \leq j^{\prime} \leq j-1} r_{j^{\prime}} \rightarrow c_{j},
$$

we have

$$
\tilde{f}_{i+y}^{n+x} \approx 2 f_{i+y}^{n+x}-\sum_{1 \leq j \leq N} s_{j}(n+x, i+y) .
$$

Thus, (23) is reduced to (11) by adding $\sum_{1 \leq j \leq N}\left(2 s_{j}+k p_{j}+(l-1) q_{j}\right)$ to both sides, and (24) is reduced to (13) by adding $\sum_{1 \leq j \leq N}\left(2 s_{j}+(k-1) p_{j}+(l+1) q_{j}\right)$ respectively. 


\section{Bäcklund Transformation for the Generalized Soliton So- lution}

We have the following proposition about a Bäcklund transformation for the generalized soliton solution.

Proposition 5 The generalized solution (5) with (7) and the following additional conditions;

$$
\begin{aligned}
& 0 \leq q_{1} \leq q_{2} \leq \cdots \leq q_{N+1} \\
& 0 \leq p_{1}-q_{1} \leq p_{2}-q_{2} \leq \cdots \leq p_{N+1}-q_{N+1},
\end{aligned}
$$

satisfies a Bäcklund transformation

$$
f_{i}^{n}+g_{i+\beta}^{n+\alpha}=\max \left(f_{i+\beta}^{n+\alpha}+g_{i}^{n}, f_{i+l+\beta}^{n-k+\alpha}+g_{i-l}^{n+k}-A\right),
$$

where $g_{i}^{n}$ is the $(N+1)$-soliton solution defined by

$$
g_{i}^{n}=\max _{\mu_{j}=0,1}\left(\sum_{1 \leq j \leq N+1} \mu_{j} s_{j}(n, i)-\sum_{1 \leq j<j^{\prime} \leq N+1} \mu_{j} \mu_{j^{\prime}} r_{j}\right)
$$

and parameters $A, \alpha, \beta$ satisfy

$$
\begin{aligned}
& A=(k-\alpha) p_{N+1}+(l+\beta) q_{N+1}, \\
& 0 \leq \alpha \leq k, \quad-k-l+\alpha \leq \beta \leq \alpha .
\end{aligned}
$$

Note that the dispersion relations (10) and (12) both satisfy the additional condition (28). Therefore the solutions given in the previous section satisfy the Bäcklund transformation (29) as a special case.

To prove Proposition [5, we rewrite $g_{i}^{n}$ by

$$
\begin{aligned}
g_{i}^{n}=\max _{\mu_{j}=0,1}( & \sum_{1 \leq j \leq N} \mu_{j} s_{j}-\sum_{1 \leq j<j^{\prime} \leq N} \mu_{j} \mu_{j^{\prime}} r_{j}, \\
& \left.\sum_{1 \leq j \leq N} \mu_{j}\left(s_{j}-r_{j}\right)+s_{N+1}-\sum_{1 \leq j<j^{\prime} \leq N} \mu_{j} \mu_{j^{\prime}} r_{j},\right) .
\end{aligned}
$$

Substituting (5) and (32) into LHS of (29), we obtain

$$
\begin{aligned}
& f_{i}^{n}+g_{i+\beta}^{n+\alpha}=\max _{\mu_{j}, \nu_{j}=0,1}\left(\sum_{1 \leq j \leq N}\left(\mu_{j}+\nu_{j}\right) s_{j}+\sum_{1 \leq j \leq N} \nu_{j}\left(\alpha p_{j}-\beta q_{j}\right)\right. \\
& \quad-\sum_{1 \leq j<j^{\prime} \leq N}\left(\mu_{j} \mu_{j^{\prime}}+\nu_{j} \nu_{j^{\prime}}\right) r_{j}, \\
& \sum_{1 \leq j \leq N}\left(\mu_{j}+\nu_{j}\right) s_{j}+s_{N+1}+\alpha p_{N+1}-\beta q_{N+1} \\
& \left.\quad+\sum_{1 \leq j \leq N} \nu_{j}\left((-k+\alpha) p_{j}-(l+\beta) q_{j}\right)-\sum_{1 \leq j<j^{\prime} \leq N}\left(\mu_{j} \mu_{j^{\prime}}+\nu_{j} \nu_{j^{\prime}}\right) r_{j}\right) .
\end{aligned}
$$


Using new parameters $\lambda_{i}$ and $\sigma_{i}$ defined by $\lambda_{j}=\mu_{j}+\nu_{j}, \sigma_{j}=-\mu_{j}+\nu_{j}(1 \leq j \leq N)$, (33) reduces to

$$
\begin{aligned}
& f_{i}^{n}+g_{i+\beta}^{n+\alpha}=\max _{\left(\lambda_{j}, \sigma_{j}\right)}\left(\sum_{1 \leq j \leq N} \lambda_{j} s_{j}+\frac{1}{2} \sum_{1 \leq j \leq N}\left(\lambda_{j}+\sigma_{j}\right)\left(\alpha p_{j}-\beta q_{j}\right)\right. \\
& \quad-\frac{1}{2} \sum_{1 \leq j<j^{\prime} \leq N}\left(\lambda_{j} \lambda_{j^{\prime}}+\sigma_{j} \sigma_{j^{\prime}}\right) r_{j}, \\
& \quad \sum_{1 \leq j \leq N} \lambda_{j} s_{j}+s_{N+1}+\alpha p_{N+1}-\beta q_{N+1} \\
& \left.+\frac{1}{2} \sum_{1 \leq j \leq N}\left(\lambda_{j}+\sigma_{j}\right)\left((-k+\alpha) p_{j}-(l+\beta) q_{j}\right)-\frac{1}{2} \sum_{1 \leq j<j^{\prime} \leq N}\left(\lambda_{j} \lambda_{j^{\prime}}+\sigma_{j} \sigma_{j^{\prime}}\right) r_{j}\right) .
\end{aligned}
$$

Similarly, we have

$$
\begin{aligned}
& f_{i+\beta}^{n+\alpha}+g_{i}^{n}=\max _{\left(\lambda_{j}, \sigma_{j}\right)}\left(\sum_{1 \leq j \leq N} \lambda_{j} s_{j}+\frac{1}{2} \sum_{1 \leq j \leq N}\left(\lambda_{j}+\sigma_{j}\right)\left(\alpha p_{j}-\beta q_{j}\right)\right. \\
& \quad-\frac{1}{2} \sum_{1 \leq j<j^{\prime} \leq N}\left(\lambda_{j} \lambda_{j^{\prime}}+\sigma_{j} \sigma_{j^{\prime}}\right) r_{j}, \\
& \quad \sum_{1 \leq j \leq N} \lambda_{j} s_{j}+s_{N+1}+\frac{1}{2} \sum_{1 \leq j \leq N} \lambda_{j}\left((-k+\alpha) p_{j}-(l+\beta) q_{j}\right) \\
& \left.\quad+\frac{1}{2} \sum_{1 \leq j \leq N} \sigma_{j}\left((k+\alpha) p_{j}-(-l+\beta) q_{j}\right)-\frac{1}{2} \sum_{1 \leq j<j^{\prime} \leq N}\left(\lambda_{j} \lambda_{j^{\prime}}+\sigma_{j} \sigma_{j^{\prime}}\right) r_{j}\right), \\
& f_{i+l+\beta}^{n-k+\alpha}+g_{i-l}^{n+k}-A=\max _{\left(\lambda_{j}, \sigma_{j}\right)}\left(\sum_{1 \leq j \leq N} \lambda_{j} s_{j}+\frac{1}{2} \sum_{1 \leq j \leq N} \lambda_{i}\left(\alpha p_{j}-\beta q_{j}\right)-A\right. \\
& +\frac{1}{2} \sum_{1 \leq j \leq N} \sigma_{j}\left((-2 k+\alpha) p_{j}-(2 l+\beta) q_{j}\right)-\frac{1}{2} \sum_{1 \leq j<j^{\prime} \leq N}\left(\lambda_{j} \lambda_{j^{\prime}}+\sigma_{j} \sigma_{j^{\prime}}\right) r_{j}, \\
& \left.\sum_{1 \leq j \leq N} \lambda_{j} s_{j}+s_{N+1}+\alpha p_{N+1}-\beta q_{N+1} \sum_{1 \leq j<j^{\prime} \leq N}\left(\lambda_{j} \lambda_{j^{\prime}}+\sigma_{j} \sigma_{j^{\prime}}\right) r_{j}\right) .
\end{aligned}
$$

Note that $\sigma_{j}$ is redefined by $\sigma_{j}=\mu_{j}-\nu_{j}$ in (35) and (36). The former argument of (34) is equal to the former of (35), and the latter to the latter of (36). Hence, (29) holds if both of the following inequalities hold for any $1 \leq N^{\prime} \leq N$.

$$
\begin{gathered}
\max _{\sigma_{j}= \pm 1}\left(\sum_{1 \leq j \leq N^{\prime}} \sigma_{j}\left(\alpha p_{j}-\beta q_{j}\right)-\sum_{1 \leq j<j^{\prime} \leq N^{\prime}} \sigma_{j} \sigma_{j}^{\prime} r_{j}\right) \\
\geq \max _{\sigma_{j}= \pm 1}\left(\sum_{1 \leq j \leq N^{\prime}} \sigma_{j}\left((-2 k+\alpha) p_{j}-(2 l+\beta) q_{j}\right)-\sum_{1 \leq j<j^{\prime} \leq N^{\prime}} \sigma_{j} \sigma_{j^{\prime}} r_{j}\right)-2 A, \\
\max _{\sigma_{j}= \pm 1}\left(\sum_{1 \leq j \leq N^{\prime}} \sigma_{j}\left((k-\alpha) p_{j}+(l+\beta) q_{j}\right)-\sum_{1 \leq j<j^{\prime} \leq N^{\prime}} \sigma_{j} \sigma_{j}^{\prime} r_{j}\right) \\
\geq \max _{\sigma_{j}= \pm 1}\left(\sum_{1 \leq j \leq N^{\prime}} \sigma_{j}\left((k+\alpha) p_{j}-(-l+\beta) q_{j}\right)-\sum_{1 \leq j<j^{\prime} \leq N^{\prime}} \sigma_{j} \sigma_{j^{\prime}} r_{j}\right)-2 \alpha p_{N+1}+2 \beta q_{N+1} .
\end{gathered}
$$


Since (37) is equivalent to (38) through the transformations $\alpha \rightarrow k-\alpha$ and $\beta \rightarrow-l-\beta$, we only need to prove (38). The maximum of LHS of (38) is given by the case of $\sigma_{j}=(-1)^{N^{\prime}-j}$, and that of RHS is given by the case of $\sigma_{N^{\prime}}=1$ and $\sigma_{j}=(-1)^{N^{\prime}-j+1}\left(1 \leq j \leq N^{\prime}-1\right)$ respectively [11]. Thus, the difference between LHS and RHS of (38) is $2\left(\alpha\left(p_{N+1}-p_{N^{\prime}}\right)-\beta\left(q_{N+1}-q_{N^{\prime}}\right)\right)$ and it satisfies

$$
2\left(\alpha\left(p_{N+1}-p_{N^{\prime}}\right)-\beta\left(q_{N+1}-q_{N^{\prime}}\right)\right) \geq 2 \alpha\left(p_{N+1}-p_{N^{\prime}}-\left(q_{N+1}-q_{N^{\prime}}\right)\right) \geq 0 .
$$

Therefore we have proved the proposition.

Next, we give the Bäcklund transformation in Type II.

Proposition 6 The generalized solution (6) with the conditions (7) and (28) satisfies the Bäcklund transformation

$$
\tilde{f}_{i}^{n}+\tilde{g}_{i+\beta}^{n+\alpha}=\max \left(\tilde{f}_{i+\beta}^{n+\alpha}+\tilde{g}_{i}^{n}-B, \tilde{f}_{i+l+\beta}^{n-k+\alpha}+\tilde{g}_{i-l}^{n+k}-A\right),
$$

where $\tilde{g}_{i}^{n}$ is the $(N+1)$-soliton solution defined by

$$
\max \left[\begin{array}{cccc}
\left|s_{1}+(-N-1) \frac{r_{1}}{2}\right| & \left|s_{1}+(-N+1) \frac{r_{1}}{2}\right| & \ldots & \left|s_{1}+(N-1) \frac{r_{1}}{2}\right| \\
\ldots & \ldots & \ldots & \ldots \\
\left|s_{N+1}+(-N-1) \frac{r_{N+1}}{2}\right| & \left|s_{N+1}+(-N+1) \frac{r_{N+1}}{2}\right| & \ldots & \left|s_{N+1}+(N-1) \frac{r_{N+1}}{2}\right|
\end{array}\right],
$$

Parameters $\alpha, \beta$ and $A$ are the same as in (31) and $B$ is defined by $\alpha p_{N+1}-\beta q_{N+1}$.

Proposition 6 is proved after the manner of Proposition 3 and Proposition 4 In particular, using a property of UP,

$$
\max \left[\begin{array}{cccc}
a_{11} & a_{12} & \ldots & a_{1 N} \\
a_{21} & a_{22} & \ldots & a_{2 N} \\
\ldots & \ldots & \ldots & \ldots \\
a_{N 1} & a_{N 2} & \ldots & a_{N N}
\end{array}\right]+\sum_{1 \leq j \leq N} b_{j}=\max \left[\begin{array}{cccc}
a_{11}+b_{1} & a_{12}+b_{1} & \ldots & a_{1 N}+b_{1} \\
a_{21}+b_{2} & a_{22}+b_{2} & \ldots & a_{2 N}+b_{2} \\
\ldots & \ldots & \ldots & \ldots \\
a_{N 1}+b_{N} & a_{N 2}+b_{N} & \ldots & a_{N N}+b_{N}
\end{array}\right],
$$

and a formula (25), $\tilde{g}_{i}^{n}$ reduces to

$$
\begin{aligned}
& \max _{\rho_{j}= \pm 1}\left(\max \left[\begin{array}{ccc}
\rho_{1}\left(s_{1}+(-N-1) \frac{r_{1}}{2}\right) & \ldots & \rho_{1}\left(s_{1}+(N-1) \frac{r_{1}}{2}\right) \\
\ldots & \ldots & \ldots \\
\rho_{N+1}\left(s_{N+1}+(-N-1) \frac{r_{N+1}}{2}\right) & \ldots & \rho_{N+1}\left(s_{N+1}+(N-1) \frac{r_{N+1}}{2}\right)
\end{array}\right]\right) \\
& =\max _{\rho_{j}= \pm 1}\left(\max \left[\begin{array}{ccc}
\rho_{1}\left(s_{1}-N \frac{r_{1}}{2}\right) & \ldots & \rho_{1}\left(s_{1}+N \frac{r_{1}}{2}\right) \\
\ldots & \ldots & \ldots \\
\rho_{N+1}\left(s_{N+1}-N \frac{r_{N+1}}{2}\right) & \ldots & \rho_{N+1}\left(s_{N+1}+N \frac{r_{N+1}}{2}\right)
\end{array}\right]-\frac{1}{2} \sum_{1 \leq j \leq N+1} \rho_{j} r_{j}\right) \\
& =\max _{\rho_{j}= \pm 1}\left(\sum_{1 \leq j \leq N+1} \rho_{j}\left(s_{j}-\frac{1}{2} r_{j}\right)-\frac{1}{2} \sum_{1 \leq j<j^{\prime} \leq N+1} \rho_{j} \rho_{j^{\prime}} r_{j}\right)+\frac{1}{2} \sum_{1 \leq j<j^{\prime} \leq N+1} r_{j^{\prime}} .
\end{aligned}
$$

Thus, we can derive through the transformation $\rho_{j}=2 \mu_{j}-1$,

$$
\tilde{f}_{i+y}^{n+x}+\tilde{g}_{i+w}^{n+z} \approx 2 f_{i+y}^{n+x}+2 g_{i+w}^{n+z}-\sum_{1 \leq j \leq N} s_{j}(n+x, i+y)-\sum_{1 \leq j \leq N+1} s_{j}(n+z, i+w) .
$$

Hence, (40) is equivalent to (29) with a difference of the negligible term $\sum_{1 \leq j \leq N} s_{j}(n, i)+$ $\sum_{1 \leq j \leq N+1} s_{j}(n+\alpha, i+\beta)$. 


\section{Relation between Bäcklund Transformations for Discrete and Ultradiscrete KdV Equations}

The $N$-soliton solution of Type II to the discrete KdV equation [5, 13,

$$
F_{i+1}^{n+1} F_{i}^{n-1}-\delta F_{i+1}^{n-1} F_{i}^{n+1}-(1-\delta) F_{i+1}^{n} F_{i}^{n}=0,
$$

is expressed by

$$
F_{i}^{n}=\left|\begin{array}{cccc}
\eta_{1}(n, i) & \eta_{1}(n+2, i) & \ldots & \eta_{1}(n+2(N-1), i) \\
\ldots & \ldots & \ldots & \ldots \\
\eta_{N}(n, i) & \eta_{N}(n+2, i) & \ldots & \eta_{N}(n+2(N-1), i)
\end{array}\right|
$$

where $\eta_{j}(n, i)$ is defined by

$$
\eta_{j}(n, i)=c_{j} \omega_{j}^{n} k_{j}^{i}+\frac{1}{c_{j} \omega_{j}^{n} k_{j}^{i}}
$$

with the dispersion relation

$$
k_{j}^{2}=\frac{1+\omega_{j}^{2} \delta}{\omega_{j}^{2}+\delta} .
$$

The Bäcklund transformations for the discrete KdV equation are expressed by

$$
\begin{aligned}
& F_{i}^{n} G_{i}^{n+1}=F_{i}^{n+1} G_{i}^{n} / D+F_{i}^{n-1} G_{i}^{n+2} / D, \\
& F_{i}^{n} G_{i-1}^{n+1}=\delta F_{i-1}^{n+1} G_{i}^{n} / D^{\prime}+F_{i-1}^{n-1} G_{i}^{n+2} / D^{\prime},
\end{aligned}
$$

where $G_{i}^{n}$ is the $(N+1)$-soliton solution,

$$
G_{i}^{n}=\left|\begin{array}{cccc}
\eta_{1}(n-2, i) & \eta_{1}(n, i) & \ldots & \eta_{1}(n+2(N-1), i) \\
\ldots & \ldots & \ldots & \ldots \\
\eta_{N+1}(n-2, i) & \eta_{N+1}(n, i) & \ldots & \eta_{N+1}(n+2(N-1), i)
\end{array}\right|
$$

and $D$ and $D^{\prime}$ are defined by

$$
D=\omega_{N+1}+1 / \omega_{N+1}, \quad D^{\prime}=k_{N+1}\left(\omega_{N+1}+\delta / \omega_{N+1}\right) .
$$

On the other hand, Proposition 6 gives the Bäcklund transformations for the uKdV equation,

$$
\begin{aligned}
\tilde{f}_{i}^{n}+\tilde{g}_{i}^{n+1} & =\max \left(\tilde{f}_{i}^{n+1}+\tilde{g}_{i}^{n}-p_{N+1}, \tilde{f}_{i}^{n-1}+\tilde{g}_{i}^{n+2}-p_{N+1}\right) \\
\tilde{f}_{i}^{n}+\tilde{g}_{i-1}^{n+1} & =\max \left(\tilde{f}_{i-1}^{n+1}+\tilde{g}_{i}^{n}-p_{N+1}-q_{N+1}, \tilde{f}_{i-1}^{n-1}+\tilde{g}_{i}^{n+2}-p_{N+1}+q_{N+1}\right),
\end{aligned}
$$

by setting $(k, l, \alpha, \beta)=(2,0,1,0),(2,0,1,-1)$ and the dispersion relation

$$
q_{j}=\min \left(p_{j}, 1\right) .
$$

In particular, we can rewrite $\tilde{f}_{i}^{n}$ and $\tilde{g}_{i}^{n}$ by

$$
\begin{gathered}
\tilde{f}_{i}^{n}=\max \left[\begin{array}{cccc}
\left|s_{1}(n, i)\right| & \left|s_{1}(n+2, i)\right| & \ldots & \left|s_{1}(n+2(N-1), i)\right| \\
\ldots & \ldots & \ldots & \ldots \\
\left|s_{N}(n, i)\right| & \left|s_{N}(n+2, i)\right| & \ldots & \left|s_{N}(n+2(N-1), i)\right|
\end{array}\right], \\
\tilde{g}_{i}^{n}=\max \left[\begin{array}{cccc}
|s(n-2, i)| & \left|s_{1}(n, i)\right| & \ldots & \left|s_{1}(n+2(N-1), i)\right| \\
\ldots & \ldots & \ldots & \ldots \\
\left|s_{N+1}(n-2, i)\right| & \left|s_{N+1}(n, i)\right| & \ldots & \left|s_{N+1}(n+2(N-1), i)\right|
\end{array}\right] .
\end{gathered}
$$


Let us discuss the ultradiscretization of (49) and its correspondence to (52). Introducing the transformations $\delta=e^{-2 / \varepsilon}, \omega_{j}=e^{p_{j} / \varepsilon}, k_{j}=e^{-q_{j} / \varepsilon}$ and $c_{j}=e^{c_{j} / \varepsilon}$, we obtain the ultradiscrete analogues of (47), (48) and (51) as

$$
\begin{gathered}
\eta_{j}(n, i)=e^{p_{j} n-q_{j} i+c_{j}}+e^{-p_{j} n+q_{j} i-c_{j}} \rightarrow\left|p_{j} n-q_{j} i+c_{j}\right|, \\
e^{-2 q_{j} / \varepsilon}=\frac{e^{\left(1-p_{j}\right) / \varepsilon}+e^{\left(-1+p_{j}\right) / \varepsilon}}{e^{\left(-1-p_{j}\right) / \varepsilon}+e^{\left(1+p_{j}\right) / \varepsilon}} \rightarrow q_{j}=\frac{1}{2}\left(\left|p_{j}+1\right|-\left|p_{j}-1\right|\right),
\end{gathered}
$$

and

$$
D \rightarrow\left|p_{N+1}\right|, \quad D^{\prime} \rightarrow-q_{N+1}+\max \left(p_{N+1},-p_{N+1}-2\right) .
$$

If $p_{j}$ 's are all positive, $q_{j}, D$ and $D^{\prime}$ in (57) and (58) are reduced to $q_{j}=\min \left(p_{j}, 1\right), p_{N+1}$ and $p_{N+1}-q_{N+1}$. However, determinants in (46) and (501) cannot be ultradiscretized directly due to the negative problem. To avoid this missing link, let us assume that ultradiscretization replaces determinant with UP. By this assumption, the ultradiscrete analogues of $F_{i}^{n}$ and $G_{i}^{n}$ are replaced with $\tilde{f}_{i}^{n}$ and $\tilde{g}_{i}^{n}$ respectively. Hence, we have the ultradiscrete analogue of (49),

$$
\begin{aligned}
& \tilde{f}_{i}^{n}+\tilde{g}_{i}^{n+1}=\max \left(\tilde{f}_{i}^{n+1}+\tilde{g}_{i}^{n}-p_{N+1}, \tilde{f}_{i}^{n-1}+\tilde{g}_{i}^{n+2}-p_{N+1}\right), \\
& \tilde{f}_{i}^{n}+\tilde{g}_{i-1}^{n+1}=\max \left(\tilde{f}_{i-1}^{n+1}+\tilde{g}_{i}^{n}-p_{N+1}+q_{N+1}-2, \tilde{f}_{i-1}^{n-1}+\tilde{g}_{i}^{n+2}-p_{N+1}+q_{N+1}\right) .
\end{aligned}
$$

The latter equation of (59) seems not to coincide with that of (52). However, in the case of $p_{N+1}>1,-p_{N+1}-q_{N+1}$ is equivalent to $-p_{N+1}+q_{N+1}-2$ by the dispersion relation. In the case of $p_{N+1} \leq 1$, we can prove

$$
\tilde{f}_{i-1}^{n+1}+\tilde{g}_{i}^{n}-p_{N+1}+q_{N+1}-2<\tilde{f}_{i-1}^{n+1}+\tilde{g}_{i}^{n}-p_{N+1}-q_{N+1} \leq \tilde{f}_{i-1}^{n-1}+\tilde{g}_{i}^{n+2}-p_{N+1}+q_{N+1}
$$

after the proof in the previous section. Thus (52) and (59) are equivalent each other.

\section{Concluding Remarks}

In this article, we consider the generalized soliton solution of two types and give the ultradiscrete soliton equations and the Bäcklund transformation. The equations are equivalent to the $\mathrm{uKdV}$ and the uToda equation in a special case. The Bäcklund transformation holds under the condition (28). The dispersion relations (10) and (12) satisfy the condition. This means that the Bäcklund transformations for the $\mathrm{uKdV}$ and the uToda equations are also obtained as a special case. Furthermore, we discuss the ultradiscretization of the Bäcklund transformations for the discrete KdV equation assuming that determinant is replaced with UP. Although determinant cannot be ultradiscretized directly in general, their counterpart gives the Bäcklund transformations for the uKdV equation.

\section{References}

[1] R. Hirota: Exact Solution of the Modified Korteweg-de Vries Equation for Multiple Collisions of Solitons, J. Phys. Soc. Japan, 33 (1972) 1456-1458.

[2] R. Hirota: Exact Solution of the Sine-Gordon Equation for Multiple Collisions of Solitons, J. Phys. Soc. Japan, 33 (1972) 1459-1463.

[3] J. Satsuma: A Wronskian Representation of $N$-Soliton solutions of Nonlinear Evolution Equations. J. Phys. Soc. Japan, 46 (1979) 359-360. 
[4] N. C. Freeman and J. J. C. Nimmo: Soliton solutions of the Korteweg-de Vreis and KadomtsevPetviashvili equations : the Wronskian technique, Phys. Lett. 95A (1983) 1.

[5] R. Hirota: Nonlinear Partial Difference Equations. I. A Difference Analogue of the Kortewegde Vries Equation, J. Phys. Soc. Japan, 43 (1977) 1424-1433.

[6] R. Hirota: Nonlinear Partial Difference Equations. IV. Bäcklund Transformation for the Discrete-Time Toda Equation, J. Phys. Soc. Japan, 45 (1978) 321-332.

[7] T. Tokihiro, D. Takahashi, J. Matsukidaira and J. Satsuma: From Soliton Equations to Integrable Cellular Automata through a Limiting Procedure, Phys. Rev. Lett. 76 (1996) $3247-3250$.

[8] J. Matsukidaira, J. Satsuma, D. Takahashi, T. Tokihiro and M. Torii: Toda-type cellular automaton and its $N$-soliton solution, Phys. Lett. A 225 (1997) 287-295.

[9] S. Tsujimoto and R. Hirota: Ultradiscrete KdV Equation, J. Phys. Soc. Japan, 67 (1998) $1809-1810$.

[10] D. Takahashi, R. Hirota: Ultradiscrete Soliton Solution of Permanent Type, J. Phys. Soc. Japan, 76 (2007) 104007-104012.

[11] H. Nagai, A New Expression of Soliton Solution to the Ultradiscrete Toda Equation, J. Phys. A: Math. Theor. 41 (2008), 235204(12pp).

[12] N. Shinzawa and R. Hirota: The Bäcklund transformation equations for the ultradiscrete KP equation, J. Phys. A: Math. Gen. 36 (2003) 4467-4675.

[13] S. Isojima, S. Kubo, M. Murata and J. Satsuma: Discrete and ultradiscrete Bäcklund transformation for KdV equation, J. Phys. A :Math. Theor. 41 (2008) 025205(8pp). 